\section{Comparação preliminar entre ultrassonografia quantitativa de falanges e densitometria óssea na avaliação da massa óssea em adolescentes}

\author{
Preliminary comparision between phalangeal \\ quantitative ultrassonography and bone densitometry \\ for bone mass evaluation in adolescents
}

Stênio Bruno Leal Duarte', Wellington Roberto Gomes de Carvalho',2, Ezequiel Moreira Gonçalves', Roberto Regis Ribeiro ${ }^{1,3}$, Edson Santos Farias ${ }^{1,4}$, Daniela de Oliveira Magro ${ }^{5}$, Laís Mariana Ribeiro de Oliveira' ${ }^{5}$, Gil Guerra-Júnior ${ }^{1}$

\section{RESUMO}

Objetivo: Avaliar associação entre ultrassonografia quantitativa de falanges da mão (QUS) e a densitometria por absorção de raio-X de dupla energia (DXA) e desses com os históricos alimentar e de fraturas. Sujeitos e métodos: Após dois anos de acompanhamento de 270 escolares, 10 com massa óssea por QUS abaixo de -2 DP foram incluídos no estudo e avaliados com DXA. Resultados: A massa óssea por DXA de L1-L4 variou de -2,8 a -1,1 DP e de corpo inteiro -2,9 e -1,2. Três estudantes apresentaram fraturas. Baixa ingestão de cálcio foi observada nos 10 casos, de fósforo em 6 e de vitamina $D$ em 8 . Não houve diferença entre os casos com massa abaixo de -2 DP nos três métodos de avaliação. Não foi observada associação entre as fraturas e o histórico alimentar, nem com os valores de massa óssea. Conclusão: Neste pequeno grupo de adolescentes houve associação entre QUS e DXA, porém sem associação entre essas avaliações e as fraturas e a ingestão de cálcio, fósforo e vitamina D. Arq Bras Endocrinol Metab. 2012;56(1):19-24

Descritores

Cálcio; DXA; ingestão dietética; massa óssea; osteossonografia

\begin{abstract}
Objective: To evaluate the association between quantitative ultrasonography at hand phalanges (QUS) and dual energy X-ray absorptiometry (DXA), and between these methods with food intake and history of bone fractures. Subjects and methods: After two years of follow up of 270 schoolchildren, 10 of them, who showed bone mass below - 2 SD in QUS, were included in the present study. Laboratory results and DXA data were analyzed. Results: Bone mass evaluated by DXA at L1-L4 ranged from -2.8 to -1.1 SDS, and whole body bone mass, from -2.9 to -1.2 SDS. Three children had history of non-pathological bone fractures. Dietary assessment showed low intake of calcium in 10 cases, of phosphorus in 6 , and of vitamin D in 8 cases. There were no differences among the cases of bone mass below -2 SD in any of the three used methods. There was no association between history of bone fractures and food intake, and between these evaluations and bone mass. Conclusion: In this small group of schoolchildren there was an association between the methods QUS and DXA. However, there was no association between bone mass and the history of bone fractures, or calcium, phosphorus and vitamin D intake. Arq Bras Endocrinol Metab. 2012;56(1):19-24
\end{abstract}

\section{Keywords}

Calcium; DXA; dietary intake; bone mass; osteosonography
1 Laboratório de Crescimento e Composição Corporal, Centro de Investigação em Pediatria (CIPED), Departamento de

Pediatria, Faculdade de Ciências Médicas, Universidade Estadual de Campinas (FCM/Unicamp), Campinas, SP, Brasil

${ }^{2}$ Instituto Federal de Educação, Ciência e Tecnologia do Sul de Minas Gerais (IFSULDEMINAS), Centro de Ciências Aplicadas à Educação e Saúde (CeCAES), Curso de Educação Física, Campus Muzambinho (MG), Muzambinho, MG, Brasil ${ }^{3}$ Departamento de Educação Física, Faculdade Assis Gurgacz (FAG), Cascavel, PR, Brasil ${ }^{4}$ Centro de Ciências da

Saúde e do Desporto (CCSD), Universidade Federal do Acre (UFAC), Rio Branco, AC, Brasil ${ }^{5}$ Departamento de Clínica Médica, Disciplina de Moléstias Infecciosas, FCM/Unicamp, Campinas, SP, Brasil

Correspondência para:

Gil Guerra-Júnior

Departamento de Pediatria, Faculdade de Ciências Médicas, Universidade Estadual de Campinas, Cidade Universitária "Zeferino Vaz" 13083-887 - Campinas, SP, Brasil gilguer@fcm.unicamp.br

Recebido em 31/Mar/2011 Aceito em 21/Dez/2011 


\section{INTRODUÇÃO}

A osteoporose deixou de ser uma preocupação exclusiva de indivíduos adultos e idosos, uma vez que a densidade mineral óssea dessa população depende da massa óssea adquirida durante os primeiros 20 a 30 anos de vida (1). A Organização Mundial da Saúde define a osteoporose como uma doença osteometabólica, caracterizada pela diminuição e deterioração da microarquitetura do tecido ósseo, com consequente aumento da fragilidade do osso e da suscetibilidade a fraturas (2).

A incidência de fraturas osteoporóticas está estreitamente relacionada à massa óssea do indivíduo, que depende tanto da quantidade de tecido ósseo adquirido durante toda a vida, em especial na puberdade e no início da vida adulta, quanto da velocidade de perda durante a vida (3). A osteoporose e as fraturas decorrentes dela são causa de elevadas taxas de morbidade, mortalidade e custos médicos em todo o mundo e, por esses motivos, têm sido consideradas um problema de saúde pública (4). A prevenção deve ser focalizada não somente na perda óssea, mas principalmente na obtenção do pico de massa óssea ideal (5), podendo este contribuir com aproximadamente $50 \%$ na variação do conteúdo mineral ósseo até a idade de 65 anos (6). Acredita-se que, ao se potencializar o acúmulo de massa óssea no período pubertário e a manutenção desse tecido na vida adulta, pode-se minimizar as reduções com o avançar da idade, contribuindo, assim, para a prevenção da osteoporose.

Assim, a saúde óssea passou a ser um tema de grande interesse científico. Ela depende de três prováveis fatores que se inter-relacionam: os níveis de hormônios circulantes que agem no processo de calcificação, a sobrecarga mecânica imposta ao esqueleto, além da ingestão e produção adequadas de cálcio e vitamina D (5). Entre os principais estudos que avaliam o papel dos nutrientes na determinação do pico de massa óssea, a ingestão dietética de cálcio recebe destaque (7-9). O reconhecimento dos fatores nutricionais conduz à possibilidade de intervenção precoce, com o intuito de prevenir o aparecimento de osteoporose e fraturas (10).

Para o estudo de massa óssea em crianças e adolescentes, a densitometria por absorção de raio $\mathrm{X}$ de dupla energia (DXA: Dual-energy $\underline{X}$-ray $\underline{\text { Absorptiome- }}$ try) é a técnica mais utilizada, porém é sabidamente dependente da densidade volumétrica e do tamanho do fragmento ósseo, trazendo limitações ao uso dessa ferramenta em crianças quando comparadas aos adultos pelas alterações no tamanho ósseo durante o crescimento $(11,12)$.

Com o desenvolvimento da osteossonografia quantitativa das falanges da mão (QUS) para estudo da massa óssea, método que utiliza como princípio a velocidade do som para analisar as propriedades quantitativas e qualitativas do material ósseo, surge uma alternativa de baixo custo e que evita a radiação ionizante em sua aplicação, que, somada ao fato de que a técnica sofre menor influência do tamanho do fragmento ósseo, torna esse um bom método a ser aplicado em pediatria para estudo da massa óssea $(12,13)$. Alguns estudos sugerem que a avaliação da massa óssea nas falanges é apropriada, uma vez que este sítio é sensível às mudanças ósseas que ocorrem durante o crescimento $(12,14)$. Entretanto, há poucos estudos na literatura que comparam a avaliação da massa óssea realizada pelo DXA e sua correlação com os resultados encontrados com QUS.

Os objetivos deste estudo foram analisar a associação entre as técnicas de QUS e DXA em estudantes de baixo nível socioeconômico, bem como a associação desses dados com o histórico de fraturas e o aporte nutricional de cálcio, fósforo e vitamina $\mathrm{D}$.

\section{CASUÍSTICA E MÉTODOS}

\section{Escolha dos sujeitos e critérios de inclusão}

Um total de 270 estudantes, entre sete e 18 anos de idade, matriculados na Escola Municipal "Professora Lydia Scalet Walker" situada no bairro Batista Genari no Município de Francisco Morato (SP), foi acompanhado desde setembro de 2008, com três avaliações de composição corporal e massa óssea: a $1^{\mathrm{a}}$ em setembro de 2008, a $2^{\text {a }}$ em março de 2009 e a $3^{\text {a }}$ em setembro de 2009. Em todas as avaliações, foram realizadas medidas de peso, altura, altura sentada, perímetro cefálico, perímetro braquial, envergadura, comprimento da mão e do pé, circunferência da cintura e do quadril, medidas de dobras cutâneas triciptal e subescapular, autoavaliação do desenvolvimento puberal (15), avaliação da massa óssea por QUS na metáfise distal de cada uma das quatro últimas falanges proximais $\left(2^{\circ}\right.$ ao $5^{\circ}$ dedo $)$ da mão não dominante realizada pelo equipamento DBM 1200 So- $^{-}$ nic BP (IGEA Carpi, Italy, $3^{\mathrm{a}}$ geração) com medidas de AD-SoS ( Amplitude Dependent $\underline{\text { Speed }} \underline{\underline{f}} \underline{\text { Sound }}$ ) (Tabela 1). A avaliação do nível socioeconômico foi realizada segundo a ABEP 2008 (16) e todos os estudantes pertenciam às classes $\mathrm{C}(28,5 \%), \mathrm{D}(58,9 \%)$ ou $\mathrm{E}(12,6 \%)$. 
Tabela 1. Dados de sexo, idade e z escore de AD-SoS de 270 escolares avaliados em três momentos distintos

\begin{tabular}{lcc}
\hline Sexo & $\begin{array}{c}\text { Masculino } \\
\mathbf{1 4 3}(\mathbf{5 3 \% )} \\
\text { Média } \mathbf{\text { DP }}\end{array}$ & $\begin{array}{c}\text { Feminino } \\
\mathbf{1 2 7}(\mathbf{4 7 \% )} \\
\text { Variação }\end{array}$ \\
\hline Idade 1 (anos) & $11,1 \pm 2,6$ & $6,7-17,9$ \\
Idade 2 (anos) & $11,6 \pm 2,6$ & $7,2-18,4$ \\
Idade 3 (anos) & $12,2 \pm 2,6$ & $7,8-19,0$ \\
Z AD-SoS 1 & $0,64 \pm 1,1$ & $-2,67-3,53$ \\
z AD-SoS 2 & $0,26 \pm 1,1$ & $-3,61-3,98$ \\
Z AD-SoS 3 & $-0,32 \pm 1,3$ & $-4,28-3,48$ \\
\hline
\end{tabular}

Após as três avaliações, entre os 270 estudantes submetidos às três avaliações sequenciais de massa óssea por QUS, apenas 10 deles apresentaram valores de $\mathrm{AD}$ -SoS, em escore de desvio-padrão (z escore), abaixo de -2 em uma das avaliações e abaixo de - 1,5 em uma segunda avaliação, conforme indicados na tabela 2 .

Esses dados foram apresentados aos estudantes $\mathrm{e}$ seus pais, e todos foram convidados a participar de uma avaliação clínico-laboratorial no Hospital de Clínicas da Unicamp. Todos os pais autorizaram seus filhos a participarem com a assinatura do Termo de Consentimento Livre e Esclarecido. O presente projeto foi aprovado pelo Comitê de Ética em Pesquisa da Faculdade de Ciências Médicas da Unicamp (Parecer CEP $\left.n^{\circ} 504 / 2009\right)$.

\section{Avaliação clínica, laboratorial e radiológica}

Esses 10 estudantes foram encaminhados para consulta no Hospital de Clínicas da Unicamp para avaliação clínica, laboratorial e radiológica. Foram feitos histó- ria clínica e exame físico completos, com avaliação de maturação sexual por um endocrinologista pediátrico, coleta de sangue para avaliação de AST, ALT, ureia, creatinina, sódio, potássio, cálcio, fósforo, fosfatase alcalina, hemograma, ferritina, anticorpo antiendomísio, T4 livre, TSH, IgFl e PTH.

A avaliação radiológica contou com a realização de raio $\mathrm{X}$ simples de punho e mão esquerda para avaliação da maturação esquelética, e DXA (Hologic ${ }^{\circledR}$, Discovery Wi 83901) dos corpos vertebrais de Ll-L4 e de corpo inteiro para confirmação da massa óssea.

\section{Avaliação nutricional}

Todos os estudantes receberam um questionário de frequência alimentar, em que foi possível fazer anotações sobre o que foi ingerido durante três dias consecutivos nas refeições principais e mesmo fora delas. Dessa forma, possibilitou-se quantificar o aporte de micronutrientes na dieta de cada escolar e realizar a comparação com as propostas de Ingestão Alimentar de Referência (Dietary Reference Intakes - DRI) do Instituto de Medicina Alimentar e da Direção de Nutrição da Academia Nacional de Ciências dos Estados Unidos (17).

Para a quantificação de cálcio, fósforo e vitamina $\mathrm{D}$ nos alimentos ingeridos, foi utilizada como base a "Tabela Brasileira de Composição dos Alimentos (TACO 2006)", desenvolvida pelo Núcleo de Estudos e Pesquisas em Alimentação da Faculdade de Engenharia de Alimentos da Unicamp e utilizada como referência para conhecimento da composição dos alimentos pelo Ministério da Saúde, bem como as informações contidas na "Tabela de Composição de Alimentos: Suporte para Decisão Nutricional” (18).

Tabela 2. Dados de idade, sexo e massa óssea de 10 escolares

\begin{tabular}{cccccccc}
\hline Caso & Sexo & Idade ${ }^{*}(\mathbf{3})$ & AD-SoS (1) & AD-SoS (2) & AD-SoS (3) & $\begin{array}{c}\text { DXA } \\
\text { L1-L4 }\end{array}$ & $\begin{array}{c}\text { DXA } \\
\text { Corpo inteiro }\end{array}$ \\
\hline 1 & M & 9,4 & $-2,67$ & $-1,58$ & $-1,74$ & $-1,1$ & $-1,2$ \\
2 & $F$ & 10,8 & $-0,46$ & $-2,49$ & $-2,07$ & $-2,2$ & $-1,9$ \\
3 & F & 10,9 & $-0,41$ & $-2,00$ & $-3,79$ & $-2,2$ & $-2,4$ \\
4 & M & 12,9 & $-1,49$ & $-2,23$ & $-3,71$ & $-2,7$ & $-2,1$ \\
5 & M & 12,1 & $-1,45$ & $-1,67$ & $-2,12$ & $-1,9$ & $-2,0$ \\
6 & M & 14,1 & $-0,53$ & $-2,24$ & $-3,28$ & $-2,8$ & $-2,3$ \\
7 & M & 15,4 & $-1,08$ & $-1,53$ & $-2,02$ & $-2,0$ & $-2,0$ \\
8 & F & 18,9 & $-1,94$ & $-2,54$ & $-2,88$ & $-2,3$ & $-2,1$ \\
9 & M & 18,2 & $-2,41$ & $-3,61$ & $-4,27$ & $-2,8$ & $-2,9$ \\
10 & M & 13,0 & $-2,07$ & $-1,35$ & $-2,33$ & $-2,1$ & $-2,2$ \\
\hline
\end{tabular}

M: masculino; F: feminino; * = em anos; ( ) = número da avaliação. 


\section{Análise estatística}

Os dados foram armazenados e avaliados utilizando-se o programa SPSS for Windows versão 13.0. Foi inicialmente realizada análise descritiva, com cálculos de média, desvio-padrão e variação (valores mínimo e máximo). Para cálculo dos micronutrientes, foi utilizado o software Dietpro 5.li profissional. Para verificar a associação entre a massa óssea por DXA e QUS, foi utilizado o teste não paramétrico pareado de Wilcoxon, e, para verificar a associação entre $\mathrm{o} z$ escore das variáveis AD-SoS, DMO em Ll-L4 e DMO de corpo inteiro em relação ao sexo, ao z da altura, à puberdade, à história de fratura óssea e aos componentes alimentares, foi utilizado o teste exato de Fisher, adotando-se sempre $\mathrm{p}<$ 0,05 como nível de significância.

\section{RESULTADOS}

Os dez estudantes ( 7 do sexo masculino), com idade cronológica variando de 9,9 a 19,4 anos, com idade óssea normal e compatível com a idade cronológica (entre 10 e 18 anos), com peso ( $z$ entre - 1,76 e 1,30 ), altura (z entre - 1,54 e 1,21) e IMC (z entre -1,23 e 1,47) normais, sendo dois pré-púberes, três em início de puberdade (estádio 2 de Tanner), dois com puberdade em andamento (estádios 3 e 4 de Tanner) e três com puberdade completa (estádio 5 de Tanner), e três com história prévia de fratura(s) em membro(s), apresentaram todos os exames laboratoriais realizados normais, incluindo o anticorpo antiendomísio, que foi negativo em todos os casos (Tabela 3).

$\mathrm{O}$ aporte de cálcio na dieta mostrou-se deficiente em todos os estudantes, variando entre $10 \%$ e $71 \%$ da ingestão recomendada. Para a vitamina $\mathrm{D}$, os valores variaram entre $13 \%$ e $106 \%$ da ingestão recomendada, sendo que apenas dois estudantes atingiram os valores mínimos recomendados. Para o fósforo, os valores variaram entre $51 \%$ e $159 \%$ da ingestão recomendada, em que quatro estudantes atingiram os valores esperados. Dos dez estudantes, três não atingiram os valores de aporte recomendados para os três nutrientes (Tabela 4).

Os valores de $\mathrm{z}$ escore de massa óssea variaram de $-4,27$ a -1,74 para AD-SoS, de -2,8 a -1,1 para DXA de Ll-L4, e de -2,9 a -1,2 para DXA de corpo inteiro. Os valores de $\mathrm{z}$ escore da $\mathrm{AD}$-SoS foram significativamente menores em relação ao DXA de Ll-L4 (p=0,013) e DXA de corpo inteiro $(\mathrm{p}=0,005)$, enquanto os valores de DXA de Ll-L4 e corpo inteiro não apresentaram di- ferenças significativas entre $\mathrm{si}(\mathrm{p}=0,082)$. Apesar desses resultados, os três métodos (QUS, DXA de L1-L4 e DXA de corpo inteiro) tiveram a mesma capacidade de identificar os casos com valores de $\mathrm{z}$ escore abaixo de -2 DP ( 8 casos). Não foi observada associação entre $z$ escore da medida massa óssea (independentemente de qual método) e sexo (Fisher $=1,00)$, $\mathrm{z}$ altura (Fisher $=1,00)$, puberdade $($ Fisher $=0,52)$, história de fratura $($ Fisher $=1,00)$, fósforo $($ Fisher $=1,00)$ e vitamina D $($ Fisher $=1,00)$ na dieta (Tabela 5$)$. Não foi observada associação entre fratura e fósforo (Fisher $=1,00)$ e vitamina $\mathrm{D}($ Fisher $=1,00)$ na dieta.

Tabela 3. Valores normais e valores encontrados dos exames laboratoriais realizados em 10 escolares

\begin{tabular}{lcc}
\hline & Valores normais & Valores encontrados \\
\hline Hemoglobina (g/dL) & $12-18$ & $12,4-14,8$ \\
Hematócrito (\%) & $36-54$ & $38,7-43,6$ \\
HCM (pg) & $27-32$ & $27,8-31,3$ \\
VCM (fL) & $80-99$ & $81,0-92,3$ \\
Leucócitos (/mm³) & $4.000-10.000$ & $7.180-9.760$ \\
Plaquetas (/mm³) & $150.000-400.000$ & $238.000-349.000$ \\
Ferritina (ng/mL) & $13-150$ & $15,1-74,9$ \\
Ureia (mg/dL) & $<50$ & $16-30$ \\
Creatinina (mg/dL) & $<0,90$ & $0,40-0,83$ \\
Sódio (mEq/L) & $136-145$ & $139-141$ \\
Potássio (mEq/L) & $3,1-5,1$ & $3,9-5,0$ \\
AST (U/L) & $<35$ & $15-27$ \\
ALT (U/L) & $<26$ & $9-20$ \\
Cálcio (mg/dL) & $9,2-11,0$ & $9,3-10,6$ \\
Fósforo (mg/dL) & $3,0-5,4$ & $3,7-5,3$ \\
Fosfatase alcalina (U/L) & $<390$ & $220-380$ \\
PTH (pg/mL) & $15-65$ & $20,7-55,6$ \\
T4 livre (ng/dL) & $0,9-1,8$ & $1,23-1,57$ \\
TSH (U/L) & $0,41-4,5$ & $1,98-4,38$ \\
IGF1 (ng/mL) & $110-700$ & $182-683$ \\
\hline & & \\
\hline
\end{tabular}

Tabela 4. Valores normais e valores encontrados na análise dos micronutrientes contidos no recordatório alimentar de três dias de oito escolares

\begin{tabular}{lcc}
\hline & Valores normais & Valores encontrados \\
\hline Cálcio $(\mathrm{mg})$ & $3000-3900$ & $382-2114$ \\
Vitamina D $(\mathrm{mcg})$ & $>15$ & $2-16$ \\
Fósforo $(\mathrm{mg})$ & $1740-3165$ & $1601-2776$ \\
\hline
\end{tabular}


Tabela 5. Frequência de valores de AD-SoS, DXA L1-L4 e DXA de corpo inteiro segundo z escore em relação ao sexo, ao z da altura, à puberdade, à história de fratura, e ao cálcio, ao fósforo e à vitamina $\mathrm{D}$ da dieta

\begin{tabular}{lccc}
\hline \multirow{2}{*}{ Sexo } & & \multicolumn{2}{c}{ z massa óssea } \\
\cline { 2 - 4 } zaltura & $\mathrm{F}$ & 3 & $>\mathbf{- 2}$ \\
& $\mathrm{M}$ & 5 & 0 \\
Puberdade & $\leq-2$ & 1 & 2 \\
& $>-2$ & 8 & 0 \\
Fratura & $1 \& 2$ & 3 & 1 \\
& $3-5$ & 5 & 2 \\
Cálcio* & + & 3 & 0 \\
& - & 5 & 0 \\
Fósforo* & + & 0 & 2 \\
& - & 6 & 0 \\
Vitamina ${ }^{*}$ & + & 2 & 4 \\
& - & 4 & 2 \\
& + & 1 & 2 \\
\hline
\end{tabular}

F: feminino; M: masculino; 1 \& 2 e 3-5: estádios de Tanner; *: dieta; + : presente; - : ausente.

\section{DISCUSSÃO}

São poucos os estudos em crianças e adolescentes normais comparando os resultados de massa óssea entre a QUS e a DXA $(12,19)$. Sundberg e cols. mostraram correlações de 0,44 e 0,70 entre QUS de calcâneo e DXA, com valores mais altos para a DXA de corpo inteiro, avaliando meninos e meninas conjuntamente (19). Halaba e cols. encontraram diferença estatisticamente significativa entre a massa óssea por QUS de falanges e a DXA de corpo inteiro e lombar apenas nos adolescentes masculinos (12). Nosso estudo, com dez escolares entre 9 e 19 anos de idade, de ambos os sexos, encontrou diferença estatisticamente significativa entre os valores de z escore da QUS de falanges e da DXA de corpo inteiro ou de coluna lombar, com valores mais comprometidos na QUS. No entanto, não houve diferença na capacidade de identificar os casos com valores de $\mathrm{z}$ escore abaixo de -2 DP nos três métodos. Esse resultado é importante, pois o método de avaliação por QUS pode significar uma vantagem na menor exposição à radiação ionizante e uma facilidade de avaliar a massa óssea fora do ambiente hospitalar.

As diferenças encontradas entre QUS e DXA são esperadas, visto que os métodos utilizam técnicas diferentes. Enquanto a QUS não depende do tamanho do osso, a DXA depende, e o tamanho do osso varia na fase de crescimento da infância e da adolescência (20). Diferentemente do esperado (21), mas possível pelo pequeno número de casos avaliados, nosso estudo não observou associação entre os valores de massa óssea pelos três métodos utilizados com o sexo, o estádio de puberdade e o $\mathrm{z}$ da estatura.

Por se tratar de uma população carente do ponto de vista socioeconômico, a avaliação clínica geral mostrando se tratar de crianças e adolescentes sem doenças e com crescimento e puberdade normais foi um dado importante para poder interpretar melhor os resultados observados na massa óssea.

O histórico de fraturas, aparentemente não patológicas, mas relacionadas a impactos de alta energia, observado em três dos dez estudantes, não apresentou associação com os valores de z escore da massa óssea por nenhum dos três diferentes métodos utilizados neste estudo, diferentemente do que se observa em adultos (22-24).

Em estudos epidemiológicos que têm o objetivo de determinar correlações entre a dieta e a presença de doenças crônicas, os métodos de análise do que é ingerido pelos participantes devem ser válidos e de baixo custo. O questionário ou recordatório da frequência alimentar é capaz de satisfazer esses dois quesitos, já tendo sido validado por diferentes estudos $(25,26)$ e, por essa razão, foi a ferramenta escolhida para este estudo.

A análise dos micronutrientes, em especial de cálcio, fósforo e vitamina $\mathrm{D}$, nesses estudantes revelou frequente e grave deficiência alimentar desses nutrientes essenciais ao crescimento e à saúde óssea. Apesar de analisar apenas o que foi ingerido em três dias, é importante avaliar que a manutenção do padrão de baixo aporte desses nutrientes por longos períodos, em especial durante a adolescência, uma das fases de maior ganho estatural e de grande formação de tecido ósseo, pode não só comprometer a estatura final do indivíduo, mas também predispor ao desenvolvimento de osteoporose na idade adulta.

Espera-se que a simples alteração de hábitos alimentares desses estudantes, com a ingestão de maior quantidade de alimentos como o leite de vaca e algum tipo de fonte proteica (especialmente as carnes), corrija as deficiências encontradas, não havendo necessidade de suplementação de micronutrientes por outras maneiras que não o aporte diário de algumas categorias de alimentos. No entanto, não devemos esquecer que essa orientação de readequação na dieta não é tarefa fácil de ser aplicada, especialmente tendo em vista o nível socioeconômico desses estudantes, nos quais é possível observar um padrão já encontrado em outros estudos de maior ingestão de alimentos de alto valor calórico e baixa quantidade de micronutrientes, que tem associa- 
ção com o desenvolvimento de sobrepeso e obesidade $(27,28)$ e/ou osteoporose (29).

Não foi observada associação entre essas deficiências nutricionais e os valores de massa óssea, e o histórico de fraturas. Esses dados, em especial a falta de associação da nutrição com a massa óssea, podem ser interpretados ou pelo número pequeno de casos avaliados, e/ou se esta deficiência nutricional não for constante e crônica, e/ou por estarmos avaliando o processo ainda na infância e adolescência e não na fase de maior incidência da osteoporose, quando esta deficiência nutricional poderá ter repercussão significativa.

Não devemos deixar de salientar as principais limitações do estudo, que foram o pequeno número de casos e a grande variação de idade e de estádio puberal entre esses casos.

Pode-se concluir que, neste pequeno grupo de estudantes com baixo nível socioeconômico, foi observada associação entre os métodos de QUS e DXA, porém não houve associação entre essas avaliações e os históricos de fraturas e ingestão de cálcio, fósforo e vitamina $\mathrm{D}$.

Agradecimentos: ao Programa Institucional de Bolsas de Iniciação Científica, Conselho Nacional de Desenvolvimento Científico e Tecnológico (PIBIC-CNPq) pela bolsa de iniciação científica (Stênio Bruno Leal Duarte), à Coordenação de Aperfeiçoamento de Pessoal de Nível Superior (Capes) pelas bolsas de doutorado (Wellington Roberto Gomes de Carvalho e Ezequiel Moreira Gonçalves) e ao CNPq pela bolsa de doutorado (Edson Santos Farias). À diretora da Escola Municipal "Professora Lydia Scalet Walker", Francisco Morato (SP), Noemi Ruiz da Silva Pereira, e ao vice-diretor, Edinaldo Antonio Milan, bem como aos alunos e responsáveis pela colaboração no estudo.

Declaração: os autores declaram não haver conflitos de interesse científico neste estudo.

\section{REFERÊNCIAS}

1. Van der Sluis IM, de Muinck Keizer-Schrama SM. Osteoporosis in childhood: bone density in children in health and disease. $J$ Pediatr Endocrinol Metab. 2001;14:817-32.

2. World Health Organization. Assessment of fracture risk and its application to screening for postmenopausal osteoporosis. WHO. 1994;843:1-129.

3. Tan LJ, Lei SF, Chen XD, Liu MY, Guo YF, Hong X, et al. Establishment of peak bone mineral density in Southern Chinese males and its comparisons with other males from different regions of China. J Bone Miner Metab. 2007;25:114-21.

4. Frazão $P$, Naveira M. Prevalência de osteoporose: uma revisão crítica. Rev Bras Epidemiol. 2006;9:206-14.

5. Heaney RP, Abrams S, Dawson-Hughes B, Looker A, Markus R, Maltrovic V, et al. Peak bone mass. Osteoporos Int. 2000;11:9851009.

6. Wu X, Hou YL, Zhang H, Shan PF, Zhao Q, Cao XZ, et al. Establishment of BMD reference databases for the diagnosis and evaluation of osteoporosis in central southern Chinese man. J Bone Miner Metab. 2008;26:586-94.
7. Abrams SA, Grusak MA, Stuff J, O'Brien KO. Calcium and magnesium balance in 9-14 y-old children. Am J Clin Nutr. 1997;66:1172-7.

8. Wang MC, Crawford PB, Hudes M, Van Loan M, Siemering K, Bachrach LK. Diet in midpuberty and sedentary activity in prepuberty predict peak bone mass. Am J Clin Nutr. 2003;77:495-503.

9. Cromer B, Harel Z. Adolescents at increased risk for osteoporosis? Clin Pediatr (Phila). 2000;39:565-74.

10. Czajka-Narins DM. Minerais. In: Mahan LK, Escott-Stump S. Alimentos, nutrição e dietoterapia. São Paulo: Roca; 2005.

11. Prentice A, Persons TJ, Cole TJ. Uncritical use of bone mineral density in absorptiometry may lead to size-related artifacts in the identification of bone mineral determinants. Am J Clin Nutr. 1994;60:837-42.

12. Halaba ZP, Konstantynowicz J, Plushiewicz W, Kaczmarshi M, Piotrawska-Jastrzebska J. Comparision of phalangeal ultrasound and dual energy $\mathrm{x}$-ray absorptiometry in healthy male and female adolescents. Ultras Med Biol. 2005;31:1617-22.

13. Falk B, Bronshtein Z, Zigel L, Constantini NW, Eliakim A. Quantitative ultrasound of the tibia and radius in prepubertal and early-pubertal female athletes. Arch Pediatr Adolesc Med. 2003;157:139-43.

14. Guglielmi G, Cammisa M, De Serio A, Scillitani A, Chiodini I, Carnevale $V$, et al. Phalangeal US-velocity discriminates between normal and vertebrally fractured subjects. Eur Radiol. 1999;9:1632-7.

15. Azevedo JCV, Brasil LMP, Macedo TBMA, Pedrosa LFC, Arrais RF. Comparação entre avaliação objetiva e autoavaliação da maturação sexual em crianças e adolescentes. J Pediatr (Rio J). 2009;85:135-42.

16. Associação Brasileira de Estudos Populacionais [Internet]. Disponível em: www.abep.or. Acesso em: 12 out 2010.

17. National Academy Press. Dietary Reference Intakes. [Internet]. Disponível em: www.nap.edu. Acesso em: 15 out 2010.

18. Philippi ST.Tabela de composição de alimentos: suporte para decisão nutricional. São Paulo: Editora Metha; 2002.

19. Sundberg M, Gärdsell P, Johnell O, Ornstein E, Sernbo I. Comparison of quantitative ultrasound measurements in calcaneous with DXA and SXA at other skeletal sites: a population-based study on 280 children aged 11-16 years. Osteoporos Int. 1998;8:410-7.

20. Rauch F, Schoenau E. Skeletal development in premature infants: a review of bone physiology beyond nutritional aspects. Arch Dis Child Fetal Neonatal Ed. 2002;86:F82-5.

21. Silva CC, GoldbergTB, Teixeira AS, Dalmas JC. A predictive analysis from bone mineral density among eutrophic Brazilian male adolescents. Arq Bras Endocrinol Metabol. 2006;50:105-13.

22. Hans D, Durosier C, Kanis JA, Johansson H, Schott-Pethelaz AM, Krieg MA. Assessment of the 10-year probability of osteoporotic hip fracture combining clinical risk factors and heel bone ultrasound: the EPISEM prospective cohort of 12,958 elderly women. J Bone Miner Res. 2008;23:1045-51.

23. Nayak S, Roberts MS, Greenspan SL. Osteoporosis screening preferences of older adults. J Clin Densitom. 2009;12:279-86.

24. Lee HD, Hwang HF, Lin MR. Use of quantitative ultrasound for identifying low bone density in older people. J Ultrasound Med. 2010;29:1083-92.

25. Wakai K. A review of food frequency questionnaires developed and validated in Japan. J Epidemiol. 2009;19:1-11.

26. Aguirre-Jaime A, De León AC, Coelho SD, Alamo CB, Carrillo-Fernandez L, Batista JCG, et al. Validación de un cuestionario de frecuencia de consumo de alimentos adaptado para el estudio $y$ seguimiento de la población adulta de las Islas Canarias. Rev Esp Salud Publica. 2008;82:509-18.

27. Moffat T.The "childhood obesity epidemic": health crisis or social construction? Med Anthropol Q. 2010;24:1-21.

28. Santos LC, Martini LA, Freitas SN, Cintra IP. Ingestão de cálcio e indicadores antropométricos entre adolescentes. Rev Nutr. 2007;20:275-83.

29. Peters BS, Martini LA. Nutritional aspects of the prevention and treatment of osteoporosis. Arq Bras Endocrinol Metabol. 2010;54:179-85. 